\title{
EVALUATION OF THE ROLE OF INTERVENTIONAL RADILOGY TECHNIQUES IN THE MANAGEMENT OF THE COMPLICATIONS OF PANCREATITIS
}

Presented by :Mohamed Adel Mohamed Gouhar, resident in radiodiagnosis department Ministry of health, university of Alexandria

Supervised by: Sherif Alsayed Hegab ,Mohsen Ahmed Abdelmohsen, Department of Radiodiagnosis,, university of

\section{Introduction}

It has been estimated that there are more than 210000 admissions for acute pancreatitis and more than 56000 hospitalizations for chronic pancreatitis in the United States each year.

The severe form of acute pancreatitis, including necrotizing pancreatitis, occurs in about $20-30 \%$ of all patients with this disease resulting in a protracted clinical course, high incidence of local complications, and high mortality rate.

In the treatment of local complications of pancreatitis no universally accepted algorithm currently exists. The approach often is dictated by the expertise of the surgeon and the interventional radiologist. The clinical status of the patient (eg, presence of sepsis, systemic inflammatory response syndrome, multisystem organ failure or acute haemorrhage) often determines the approach to be taken.

Image-guided drainage procedures have proved to be effective alternatives to surgery, particularly early in the course of

complications from severe acute pancreatitis with necrosis. Some of these percutaneous procedures are performed to stabilize seriously ill patients before surgery (bridge care), and others are intended to cure. Interventional radiology also is called for ancillary procedure.

Pseudoaneurysms or active bleeding related to acute pancreatitis are usually diagnosed on the basis of contrast enhanced CT findings, and images should be obtained in late arterial and portal venous phases.

\section{AIM OF THE WORK}

The aim of this study is to assess the role of intervention radiology in the management of pancreatitis and its complications Alexandria

\section{METHODS}

The studied patients were subjected to the following:

- Written informed consent. History taking. Clinical examination.

- Pre and post ultrasound/ Multidetector CT scanner to assess the presence of the pancreatitis and its complications.

- Interventional radiology procedures were performed according to the clinical context.

- Image-guided (US/CT) drainage/ with catheter insertion.

- Image-guided embolization for bleeding vessels and pseudoaneurysms as complication of pancreatitis.

- Post procedure evaluation and follow up.
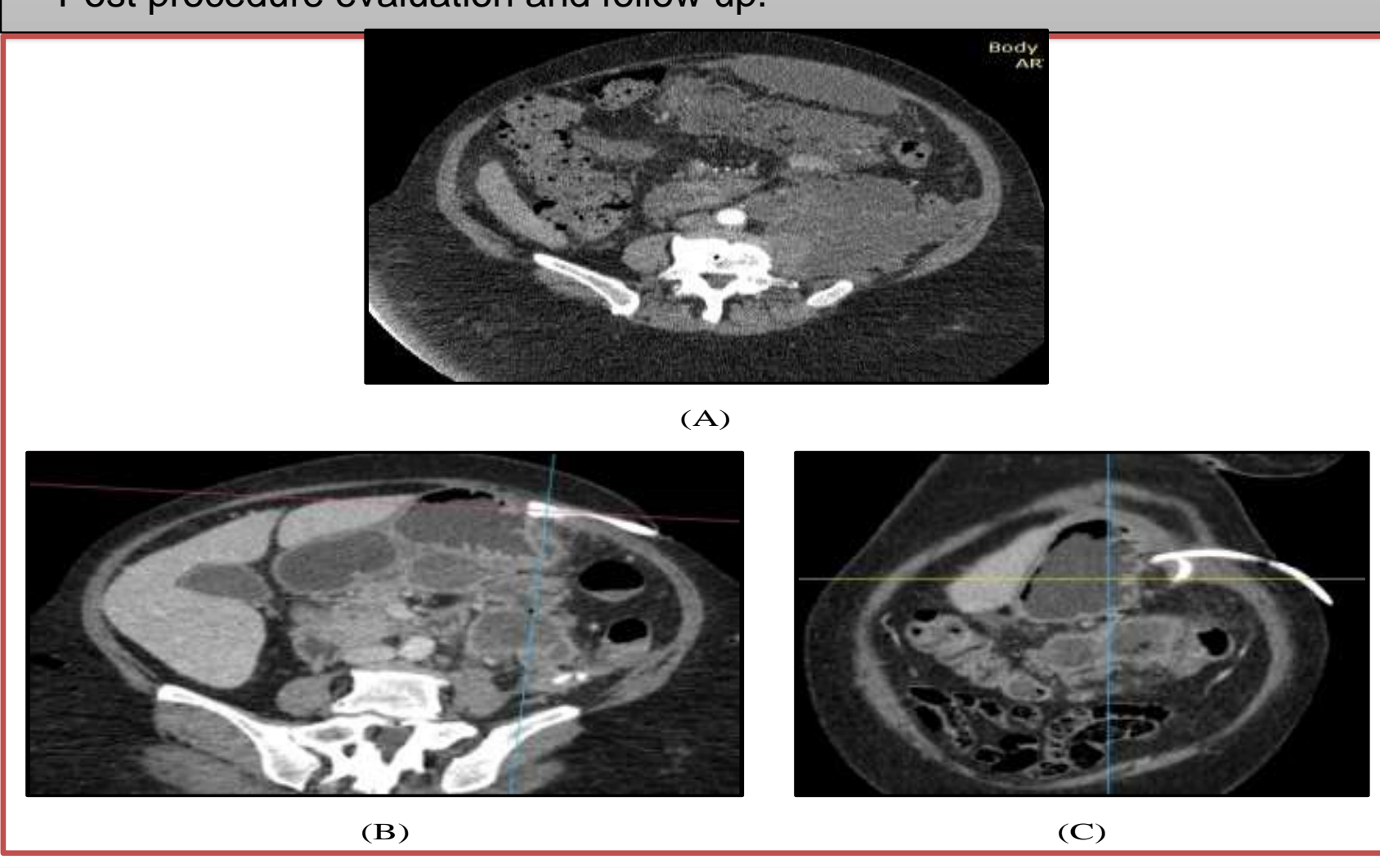

(A) Axial cut of CECT showed Multipleperi-pancreatic collections, at the properitoneum, left para-colic gutter and left sided retro- peritoneum. (B \& C) Two pig tail catheters inserted and the collections were nearly drained.

\section{RESULTS}

The study included 20 patients referred to the Radiology Department of Alexandria Main University Hospital, Faculty of Medicine, Alexandria University aiming to interventional radiological management of complications sequel to pancreatitis.

- The patients had a variety of complications sequel to the pancreatitis. 6 patients presented by large pancreatic psoducyst $(>5 \mathrm{~cm}) .12$ patients presented by varieties of peripancreatic fluid collections. 2 patients presented by splenic artery pseudoaneurysm.

- The majority of our interventions was ultrasound guided, 15 out of 20 patients about $75 \%$ of our cases managed by ultrasound guided drainage and pig tail catheter insertion. Two patients needed the help of fluoroscopic guided technique along about $10 \%$. And only one case was done CT guided.

We have two cases of splenic artery pseudoaneurysms managed by angiography and embolization.

We had clinical and technical success in all the performed procedures except for one patient, who was a patient had a huge pseudocyst with underlying pancreatic duct injury, underwent to surgery and our intervention was like a bridge therapy.

\section{CONCLUSION}

Interventional radiology plays an increasing crucial role in the multidisciplinary management of complications pancreatitis. It provides a minimally invasive therapy also in critical patients, reducing recovery times and avoiding operation morbidity.

Some of these percutaneous procedures are performed to stabilize seriously ill patients before surgery (bridge care), and others are intended to cure.

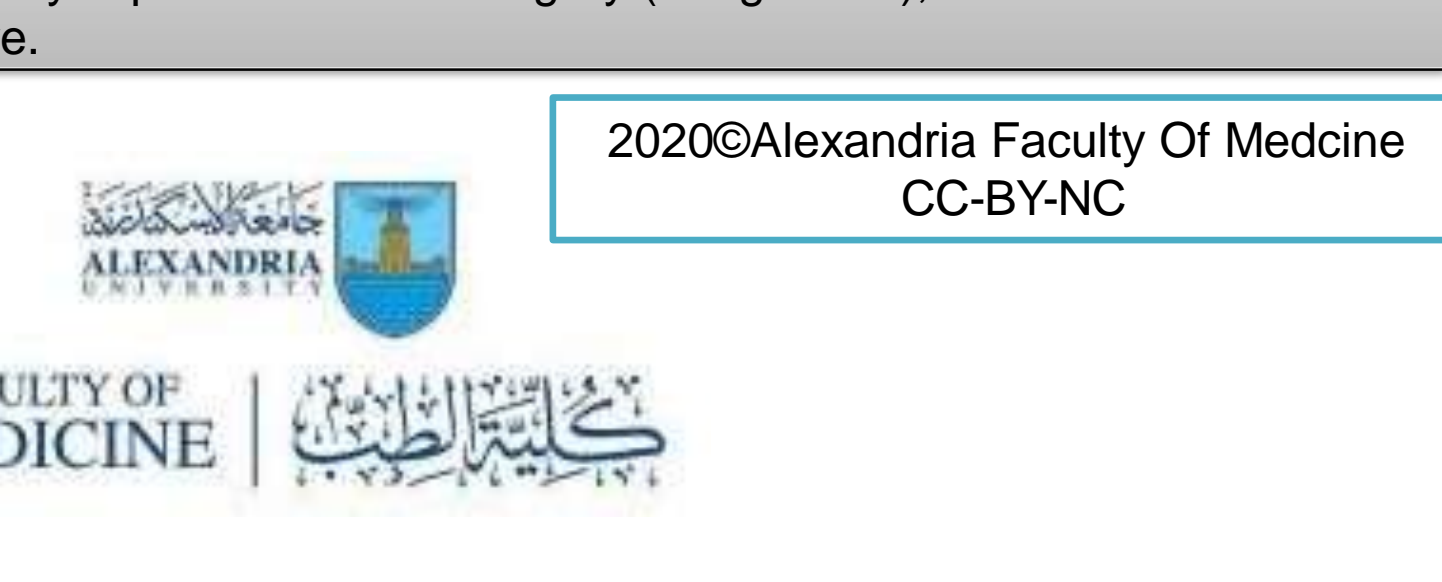

\section{PATIENTS}

The study was conducted on 20 patients suffering from pancreatitis and/or one of its complications presenting to the intervention radiology unit in Alexandria Main University hospital and Elmowasah university hospital.

pro-

